\title{
Covalent chemistry on a van der Waals heterostructure
}

Julia Villalva, † Sara Moreno-Da Silva, † Palmira Villa, ¥ Luisa Ruiz-González,§ Cristina Navío, † Saül García-Orrit, † Víctor Vega-Mayoral, † Juan Cabanillas-González*, † Andrés Castellanos-Gómez, • Emerson Giovanelli, $†$ and Emilio M. Pérez ${ }^{\star} \dagger$

† IMDEA Nanociencia; c/ Faraday 9, Campus de Cantoblanco; 28049 Madrid, Spain.

‡ Instituto Pluridisciplinar; Universidad Complutense de Madrid; Paseo de Juan XXIII No 1 , 28040 Madrid, Spain.

§ Departamento de Química Inorgánica; Facultad de ciencias químicas, Universidad Complutense de Madrid, Av. Complutense s/n, 28040 Madrid, Spain.

- Instituto de Ciencia de Materiales de Madrid (ICMM-CSIC), Sor Juana Inés de la Cruz, 3, Campus de Cantoblanco, 28049 Madrid, Spain.

Keywords: van der Waals heterostructures $\bullet$ thiol-ene $\bullet$ click chemistry $\bullet$ maleimides $\bullet 2 \mathrm{D}$ materials

\section{Abstract}

The building of van der Waals heterostructures ${ }^{1-7}$ and the decoration of $2 \mathrm{D}$ materials with organic molecules $^{8-10}$ share a common goal: to obtain ultrathin materials with tailored properties. ${ }^{11-17}$ Performing controlled chemistry on van der Waals heterostructures would add an extra level of complexity, providing a pathway towards 2D-2D-0D mixed-dimensional heterostructures. Here we show that thiol-ene-like "click" chemistry ${ }^{18,} 19$ can be used to decorate franckeite, ${ }^{20-23}$ a naturally occurring van der Waals heterostructure with maleimide reagents. ATR-IR and NMR analyses corroborate the Michael addition mechanism via the formation of a $\mathrm{S}-\mathrm{C}$ covalent bond, while Raman and HR-TEM show that the $\mathrm{SnS}_{2}$-PbS alternating structure of franckeite is preserved, and suggest that $\mathrm{SnS}_{2}$ reacts preferentially, which is confirmed through XPS. We illustrate how this methodology can be used to add functional molecular moieties by decorating franckeite with porphyrins. UV-vis-NIR spectroscopy confirms that the chromophore ground state remains operative, showing negligible ground-state interactions with the franckeite. Excited-state interactions across the hybrid interface are revealed. Time-resolved photoluminescence confirms the presence of excited-state de-activation in the linked porphyrin ascribed to energy transfer to the franckeite.

\section{Introduction}

The stack of different 2D materials is the root of the field of van der Waals heterostructures. ${ }^{1-3}$ The most widespread method for the synthesis of heterostructures of bidimensional materials is the direct growth of one material on top of the other by CVD, or van der Waals ${ }^{4}$ or edge ${ }^{5}$ epitaxy. Alternatively, one can manually stack one material on top of the other, using deterministic transfer 
methodologies. ${ }^{6,7}$ This is usually done by using a viscoelastic polymer as transfer media, on which one of the flakes is adhered and then placed on top of the other using optical microscopy and spectroscopy to monitor the alignment. ${ }^{24}$ Alternatively, one can obtain ultrathin van der Waals heterostructures from the direct exfoliation of minerals of adequate composition. ${ }^{20-23,25-28}$ The motivation behind this field of research is based on the expectation that the combination (or modulation) of properties of nanomaterials is a promising approach towards materials by design. ${ }^{11-17}$ This exact same motivation fuels the interest in the chemistry of $2 \mathrm{D}$ materials: ${ }^{8,9}$ we expect that the decoration of the nanomaterials with functional molecular fragments will yield superior combined properties. ${ }^{10}$ Besides this application-oriented point of view, the chemistry of 2D materials presents very interesting fundamental challenges, like obtaining chemical selectivity in materials where most atoms are chemically identical. ${ }^{29}, 30$ To the best of our knowledge, the covalent chemistry of van der Waals heterostructures is still unexplored territory.

With these considerations in mind, we decided to explore the chemistry of a naturally occurring van der Waals heterostructure: franckeite. Franckeite (Fk) is composed of the alternate layers of one pseudohexagonal $(\mathrm{H})$ tin(IV) sulfide $\left(\mathrm{SnS}_{2}\right)$-like layer and four pseudotetragonal $(\mathrm{T})$ lead(II) sulfide (PbS)-like layers (Figure 1a). ${ }^{31}$ Among its attractive features, we can highlight that Fk is an airstable semiconductor with a very small bandgap $(0.5-0.7 \mathrm{eV})$ and presents $p$-type doping, mostly due to substitutional $\mathrm{Sb}(\mathrm{III})$ in the structure. ${ }^{32}$

We have very recently reported the use of thiol-maleimide "click" chemistry ${ }^{18,19}$ to functionalize $\mathrm{MoS}_{2}$ and $\mathrm{WS}_{2}$ under particularly mild conditions. ${ }^{33}$ We believed this powerful reaction would be an ideal tool to take the first steps towards heterostructures of the 2D-2D-0D type, where the $0 D$ material are functional organic molecules. From the fundamental chemistry point of view, we wanted to address the possibility of performing thiol-maleimide chemistry on a material composed of two different sulfides.

Here, we describe how to functionalize Fk covalently using maleimides as soft electrophiles. At room temperature in isopropanol ( $\mathrm{PrOH})$, we achieve preferential reactivity of the sulfides in the $\mathrm{SnS}_{2}$ layer to form S-C bonds, with sufficient selectivity to conserve the van der Waals heterostructure. As proof-of-principle application, we describe the functionalization of Fk with a porphyrin-decorated maleimide.

\section{Results and discussion}


a)

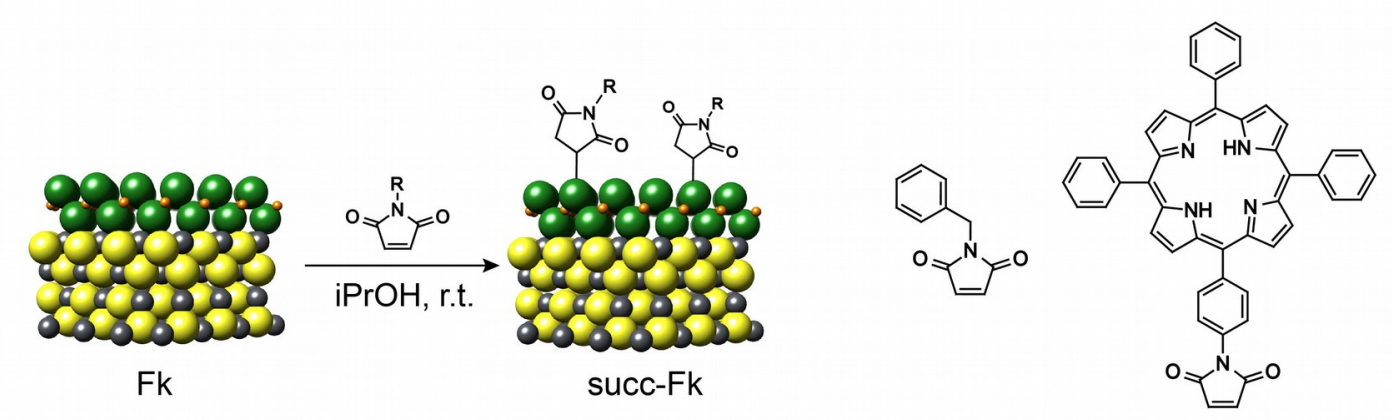

b)

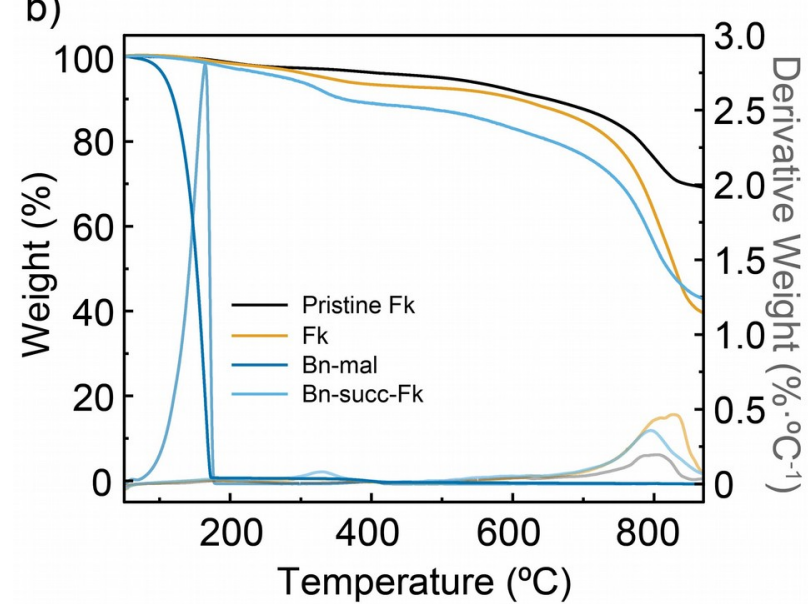

c)

Bn-mal
TPP-mal

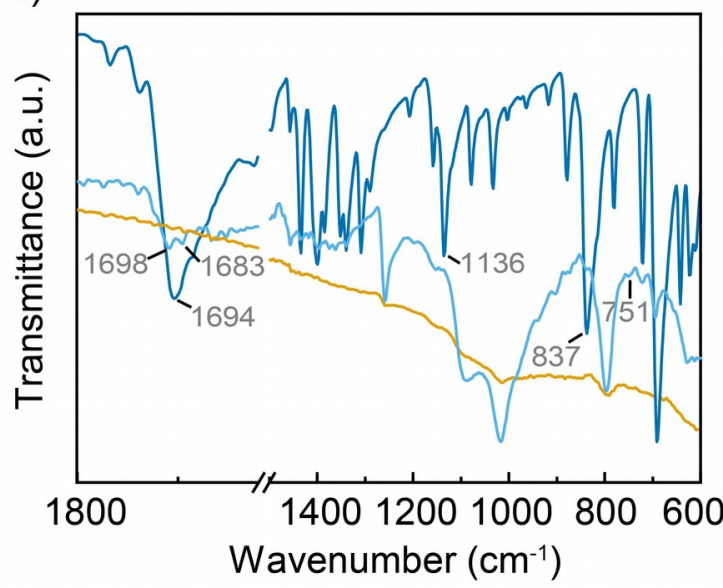

d)

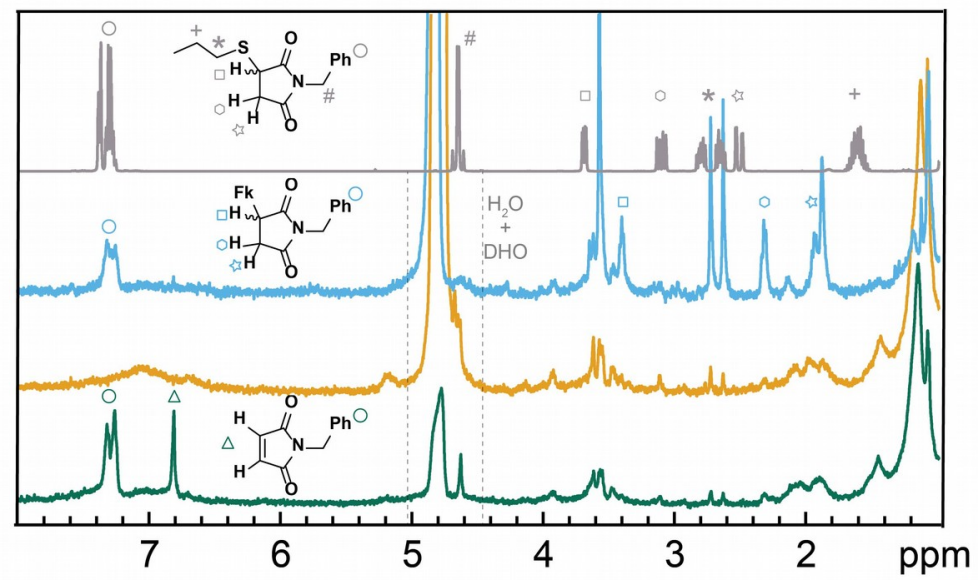

Figure 1. a) Chemical structure of Fk from its X-ray crystal structure, ${ }^{31}$ and idealized reaction scheme with maleimides. Based on HR-TEM and XPS data we show $\mathrm{SnS}_{2}$ as the reactive layer. The $\mathrm{S}$ atoms in the $\mathrm{SnS}_{2}$-like layers are green, $\mathrm{S}$ atoms in the PbS-like layer are yellow, $\mathrm{Sn}$ atoms are orange and $\mathrm{Pb}$ atoms are gray. The structures of the two maleimide reagents are also shown. b) TGA $\left(\mathrm{N}_{2}, 10^{\circ} \mathrm{C} \cdot \mathrm{min}^{-1}\right)$ of pristine exfoliated Fk (black), control Fk (yellow), Bn-maleimide (intense blue) and functionalized Bn-succ-Fk (pale blue). The first derivatives are shown in thinner lines. c) Comparison of the ATR IR spectra of control Fk, Bn-maleimide and functionalized Bn-succ-Fk (same colour code as in b). d) HR-MAS-1H-NMR (500 MHz) spectra of Bn-succ-Fk (pale blue), 
control Fk (orange) and a mixture of Fk and Bn-maleimide (dark green) obtained using $\mathrm{D}_{2} \mathrm{O}$ as the reference. The solution ${ }^{1} \mathrm{H} \mathrm{NMR}\left(\mathrm{CDCl}_{3}, 400 \mathrm{MHz}\right.$, r.t.) of the product of the reaction between 1propanethiol and $\mathrm{N}$-benzylmaleimide is also shown for comparison (gray).

The structure of Fk and the idealized reaction scheme are shown in Figure 1. For simplicity, only a minimal repetition unit is drawn. Few-layer Fk colloids were obtained using the liquid-phase exfoliation method previously described. ${ }^{34}$ Briefly, Fk powder obtained from careful grinding of mineral pieces was bath ultrasonicated in $\mathrm{iPrOH}\left(10 \mathrm{mg} \cdot \mathrm{mL}^{-1}\right)$ at $20^{\circ} \mathrm{C}$ for $1 \mathrm{~h}$. The colloid obtained in the supernatant after centrifugation at $988 \mathrm{~g}$ for $30 \mathrm{~min}$ presented a homogeneous distribution of flakes, most of them having a few-layer thickness ( $<7.5 \mathrm{~nm}$ from AFM data, S4). To this colloid, $N$ benzylmaleimide (Bn-mal in Figure 1) was added, and the mixture was ultrasonicated for 5 min then stirred overnight. As for the purification procedure, the suspension was filtered through a polytetrafluoroethylene membrane with a pore size of $0.2 \mu \mathrm{m}$, and the solid was washed several times with $\mathrm{CHCl}_{3}$ to remove excess reagents and physisorbed maleimide, $\mathrm{CHCl}_{3}$ was selected after an optimization process as the best washing solvent due to its ability to dissolve Bn-mal. The purification process was monitored by UV-Vis spectroscopy (see S5).

Using thermogravimetric analysis (TGA) the functionalization was estimated at ca. 7 wt.\% for the $\mathrm{Bn}$-mal and ca. 4 wt.\% when the porphyrin-derived maleimide (TPP-mal) was employed (Figure $1 \mathrm{~b}$ and S6). For a rigorous analysis, a control Fk sample was stirred overnight and rinsed afterwards with $\mathrm{CHCl}_{3}$ following the exact same procedure described for the functionalization. This control sample presents an initial weight loss at around $300{ }^{\circ} \mathrm{C}$ which can correspond to adsorbed solvent; then it is thermally stable until $700^{\circ} \mathrm{C}$, where it starts to decompose. The organic material loss overlaps that of the solvent but is displaced to much higher temperatures (from $155^{\circ} \mathrm{C}$ to $310^{\circ} \mathrm{C}$ in Bn-succ-Fk and from $460{ }^{\circ} \mathrm{C}$ to $560^{\circ} \mathrm{C}$ in TPP-succ-Fk). This improved thermal stability is a first indication that the organic material is not simply physisorbed onto the surface but forming covalent bonds. The low degree of functionalization compared to $\mathrm{MoS}_{2}$ and $\mathrm{WS}_{2}$ under similar reaction conditions $^{33}$ is rationalized as a direct consequence of the structure of $\mathrm{Fk}$ (see below).

ATR-FTIR shows the presence of new vibration bands in the Bn-succ-Fk sample (Error: Reference source not foundc). The intense carbonyl stretch that appears at $1694 \mathrm{~cm}^{-1}$ in the Bn-mal splits into two new bands in the functionalized sample $\left(1698\right.$ and $\left.1683 \mathrm{~cm}^{-1}\right)$. This can be explained by the desymmetrization of the molecule after functionalization. Additionally, the intense alkene $\mathrm{C}-\mathrm{H}$ bending mode at $837 \mathrm{~cm}^{-1}$ is strongly depleted after functionalization, which is in accordance with the expected Michael addition mechanism. We assign the new $751 \mathrm{~cm}^{-1}$ weak band, absent in the reference $\mathrm{Fk}$ material and in $\mathrm{Bn}-\mathrm{mal}$, to the new $\mathrm{C}-\mathrm{S}$ stretching vibration. A similar displacement has been observed for the $\mathrm{C}-\mathrm{S}$ bond in different $2 \mathrm{D}$ materials such as $\mathrm{MoS}_{2}$ and $\mathrm{WS}_{2}{ }^{33}$ As a check, a mixture of the $\mathrm{N}$-benzylmaleimide derivative, the $\mathrm{N}$-benzylsuccinimide and $\mathrm{Fk}$ was stirred under 
the typical reaction conditions. In this case, no organic signals are observed in FT-IR after performing the same number of washes as for Bn-succ-Fk (see SI, S7) indicating that the reaction requires the double bond from the $N$-benzylmaleimide to work. Moreover, it also implies that the washing procedure is efficient to remove the physisorbed organic molecules.

Conclusive evidence about the functionalization and the functionalization mechanism is obtained when the powder sample is analysed using High-Resolution Magic Angle Spinning (HR-MAS) NMR. HR-MAS ${ }^{1} \mathrm{H}$ NMR spectroscopy permits the direct analysis of heterogeneous samples, such as tissue $^{35}$ or materials, ${ }^{36}$ and has previously been used to track organic reactions in the solid state. ${ }^{37}$ Using deuterated water as the reference, the control Fk sample (orange in Error: Reference source not found) shows only weak signals in the alkane region, probably due to adsorbed hydrocarbons and residual $\mathrm{iPrOH}$, and a broad a very weak signal centered around $7 \mathrm{ppm}$ (most likely physisorbed $\mathrm{iPrO} \underline{\mathrm{H}})$. In contrast, the benzylsuccinimide signals are clearly observed in the functionalized Bn-succ-Fk (blue in Error: Reference source not found), which can be unambiguously assigned to covalent functionalization of Fk through Michael addition by comparison with a Fk and $N$-benzylmaleimide mixture (green in Error: Reference source not found): the total depletion of the alkene signals at around $6.8 \mathrm{ppm}$ (green triangle, ca. $6.8 \mathrm{ppm}$ ) is accompanied by the appearance of three new signals (marked with a square at ca. $3.4 \mathrm{ppm}$, an hexagon at ca. $2.3 \mathrm{ppm}$, and star at ca. $1.9 \mathrm{ppm}$, in Error: Reference source not found) in the alkane region. The new signals correspond to the new aliphatic diastereotopic protons of the newly formed succinimide. Comparison with the experimental ${ }^{1} \mathrm{H}$ solution NMR spectrum of the product of the reaction between 1-propanethiol and $\mathrm{N}$-benzylmaleimide $\left(\mathrm{CDCl}_{3}, 400 \mathrm{MHz}\right.$, r.t., gray in Error: Reference source not found), where the signals can be unambiguously assigned, further confirms this observation: a consistent upfield shift is observed for all the succinimide signals due to the presence of Fk, while the aromatic protons, initially oriented away from Fk, are hardly affected. Unfortunately, the benzylic $-\mathrm{CH}_{2}$ cannot be reliably identified in the different Fk samples, as spectra were acquired with water suppression.

With the reaction effectiveness and mechanism clearly established, we went on to investigate if/how the Fk structure and electronic properties were affected by the covalent modification. To this end, Raman spectroscopy provided valuable information. Both control and functionalized samples exhibit very similar spectra, with the characteristic Fk Raman peaks (Figure S8 and Table S9). In particular, we observe a band centered at $86 \mathrm{~cm}^{-1}$, which results from the spheroidal and transverse acoustic phonon modes of the PbS layers. The band at $141 \mathrm{~cm}^{-1}$ is assigned to a combination of the $2^{\text {nd }}$ order effect of the $\mathrm{SnS}_{2}$ layers and the transverse optical and acoustic phonon modes of the $\mathrm{PbS}$ layers. The outcome of the longitudinal optical phonon mode of the PbS layers and the atomic orbital $E_{g}$ of the $S_{n} S_{2}$ layers is the band at $201 \mathrm{~cm}^{-1}$. The band at $253 \mathrm{~cm}^{-1}$ is the combination of the phonon modes of both layers, $\mathrm{PbS}$ and $\mathrm{SnS}_{2}$, and therefore its relative intensity is a good indication 
that the thickness of the unique 2D-2D heterostructure is preserved upon functionalization, which is as well proven by AFM (see SI, S10). The $A_{1 g}$ mode of $\mathrm{SnS}_{2}$ results in the formation of the band at $318 \mathrm{~cm}^{-1}$. Finally, the shoulder from $\sim 400$ to $650 \mathrm{~cm}^{-1}$ shows the longitudinal optical phonon modes of $\mathrm{PbS}$ and a $2^{\text {nd }}$ order effect from the $\mathrm{SnS}_{2}$ layers. ${ }^{20}$
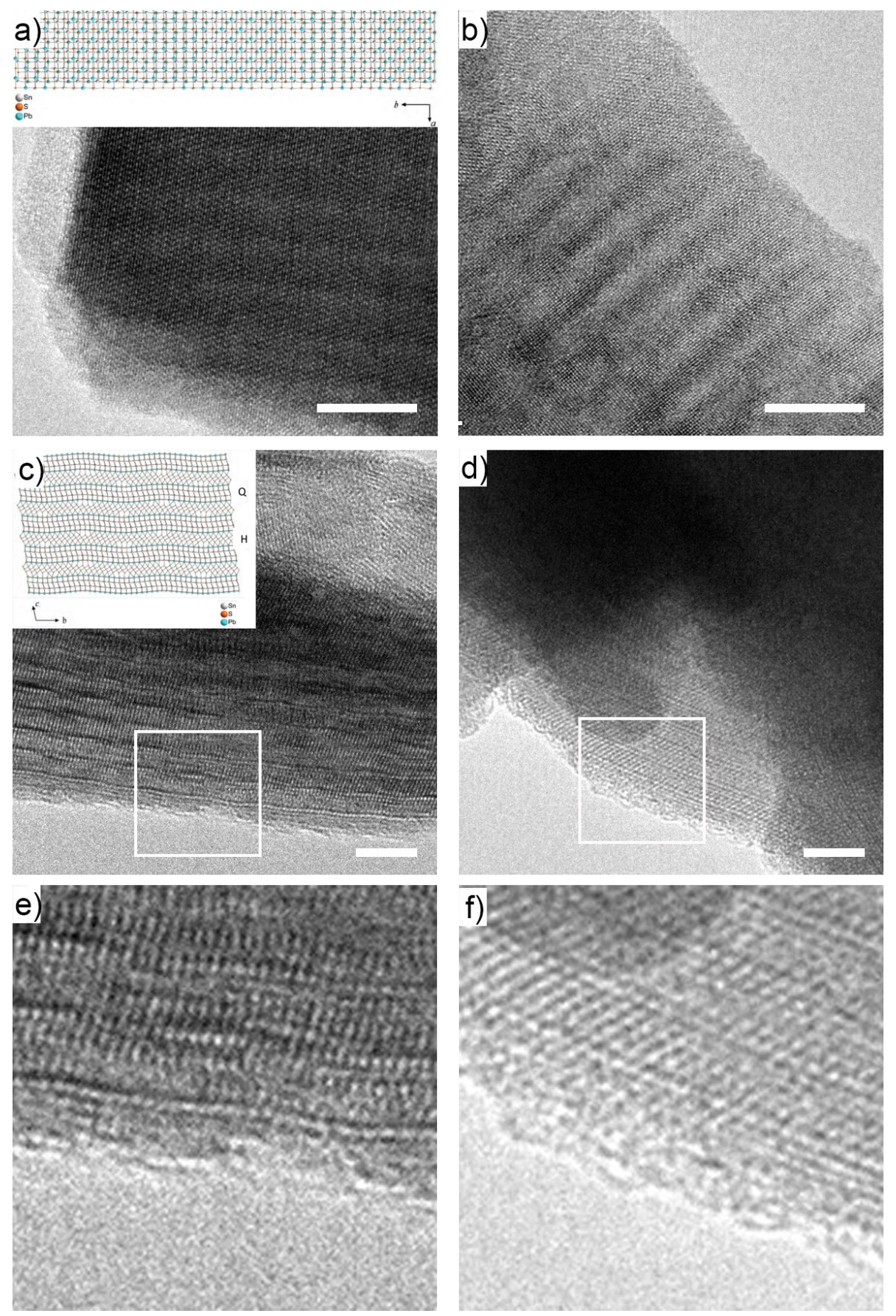

Figure 2. HR-TEM micrographs obtained for pristine Fk (a, c and e) and Bn-succ-Fk (b, d and f). The insets show the orientations that originate the Moiré pattern in $a$ an $b$, and the edge-on 
perspective of $c$ and $d .{ }^{31}$ Scale bars are $10 \mathrm{~nm}$ for $a$ and $b$ and $5 \mathrm{~nm}$ for $c$ and $d$. e) and f) show a zoom in on the edge areas highlighted with white squares in c) and d), respectively.

In agreement with the Raman measurements, high-resolution transmission electron microscopy HRTEM $(200 \mathrm{kV})$ shows no major changes in the van der Waals heterostructure during functionalization. Figure $a$ and $b$ show the comparison of characteristic micrographs for Fk and Bnsucc-Fk along the [100] direction (see inset). ${ }^{31}$ In both micrographs, the same Moiré pattern is observed, which originates from the superposition of the $\mathrm{H}\left(\mathrm{SnS}_{2}\right)$ and $\mathrm{Q}(\mathrm{PbS})$ layers (see SI, S11 for additional measurements). Figure $c$ and $d$, correspond to an edge-on perspective of both samples. The measured periodicities, as better observed in the enhanced details (Figure e and f), are in agreement with the stacking of the $\mathrm{H}$ and $\mathrm{Q}$ layers of the Fk lattice. A closer look at the final layer (Figure e and $\mathrm{f}$ ) suggests that the $\mathrm{H}$ layer, i.e., $\mathrm{SnS}_{2}$, as proved by Velicky et al. ${ }^{[10]}$, is the one exposed to ambient and is therefore most probably the one that reacts preferentially. This is consistent with the chemical intuition based on the lower electronegativity of $\mathrm{Sn}$ compared to $\mathrm{Pb}$, and the smaller radius and higher charge of $\mathrm{Sn}(\mathrm{IV})$ compared to $\mathrm{Pb}(\mathrm{II})$ : the combination of these properties should render $\mathrm{SnS}_{2}$ more purely ionic compared to $\mathrm{PbS}^{38}$ and, as a consequence, the sulfur atoms directly attached to Sn more anionic and more nucleophilic. This hypothesis also explains the low degree of functionalization observed, as for every potentially reactive $\mathrm{SnS}_{2}$ layer, there are four PbS layers that remain fundamentally intact. Additionally, the amorphous layer due to organic material appears thicker in Bn-succ-Fk, in accordance with functionalization, although this last observation is far from being conclusive.

To further investigate this point, we have performed $X$ ray Photoelectron Spectroscopy (XPS) measurements under Ultra High Vacuum (UHV) conditions (see SI for experimental details). The C 1s core level centered in $284.6 \mathrm{eV}$ was used as a binding energy reference and the selected regions of $\mathrm{S} 2 \mathrm{p}, \mathrm{Pb} 4 \mathrm{f}$ and $\mathrm{Sn} 3 \mathrm{~d}$ are shown in Figure 3. With the known structure of Fk, we would expect for the valence states of the pristine material to show two different chemical environments for $\mathrm{S}$ (one related to the $\mathrm{Q}$ type and another to the $\mathrm{H}$ type), one for the $\mathrm{Pb}$ (related to the $\mathrm{Q}$ layer) and two for the $\mathrm{Sn}$ (one related to the $\mathrm{Q}$ type and another to the $\mathrm{H}$ type). In Figure a-c we show the core levels for the $S 2 p, P b 4 f$ and $S n 3 d$ for the Fk control sample. The fit of the $S 2 p$ region shows two clear doublets with the $2 p_{3 / 2}$ level centered at $160.5 \mathrm{eV}$ and $161.5 \mathrm{eV}$. The proportion of the area for each doublet can help on the labeling of the components: as the $\mathrm{Q}$ layer is composed by four atomic layers with $S$, the intensity of this component in the $S 2 p$ core level is expected to be larger than for the $\mathrm{H}$ layer. We therefore assign the component at $160.5 \mathrm{eV}$ to the $\mathrm{Q}$ layer and the one centered in $161.5 \mathrm{eV}$ to the $\mathrm{H}$ layer. For the $\mathrm{Pb} 4 \mathrm{f}$ case (Figure b) we observe two chemical states instead of one, the $\mathrm{Pb}^{2+}$ expected for the $\mathrm{Q}$ layer and the appearance of a $\mathrm{Pb}^{4+}$ component 
that can be attributed to the appearance of lead oxide. ${ }^{20}$ For the $\mathrm{Sn} 3 \mathrm{~d}$ we only observe one chemical state for the Fk control, which shows the Sn 3d5/2 centered at $485.8 \mathrm{eV}$.

After functionalization we observe some changes on the XPS spectra, shown in Figure d-f. Due to the formation of a $\mathrm{C}-\mathrm{S}$ bond, a third component could be expected in the $\mathrm{S} 2 \mathrm{p}$ region, but the low degree of functionalization together with limitations on the resolution of XPS make this new chemical state overlap with the components for the Fk control. ${ }^{39}$ This is reflected as a change in the relative atomic percentage in the area of the $S 2 p$. The evidence of the functionalization in the XPS spectra (apart from the appearance of a very weak $N$ 1s peak) is the new component in the Sn $3 d$ region (Figure 3f) located in $487.4 \mathrm{eV}$, which amounts to $7 \%$ of the total area, in good agreement with the degree of functionalization determined by TGA, and is again in support of preferential reaction of the $\mathrm{SnS}_{2}$ layers. 
a)

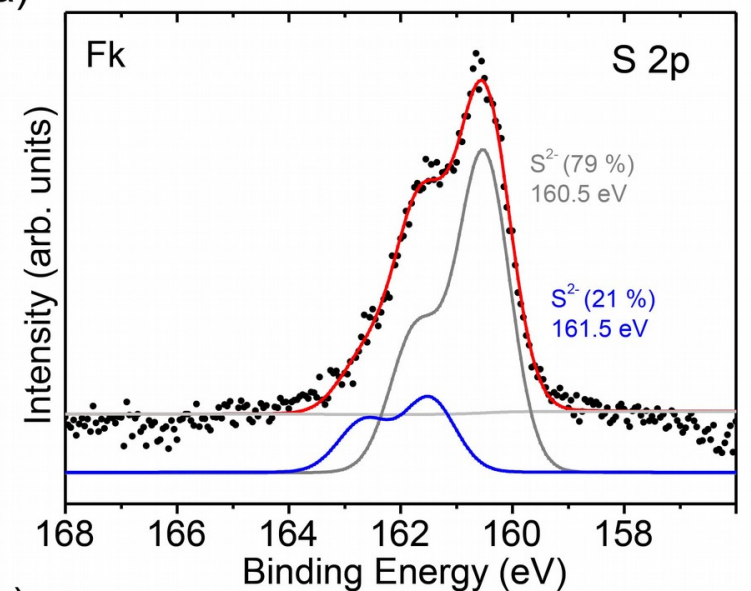

c)

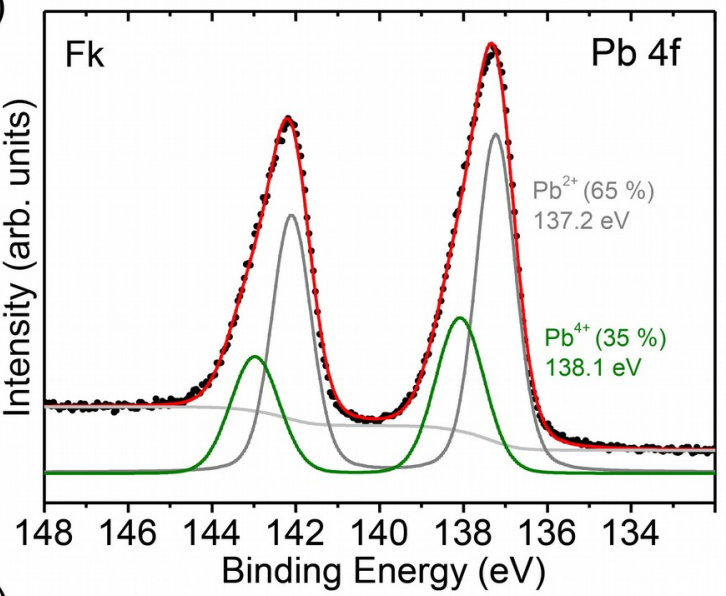

e)

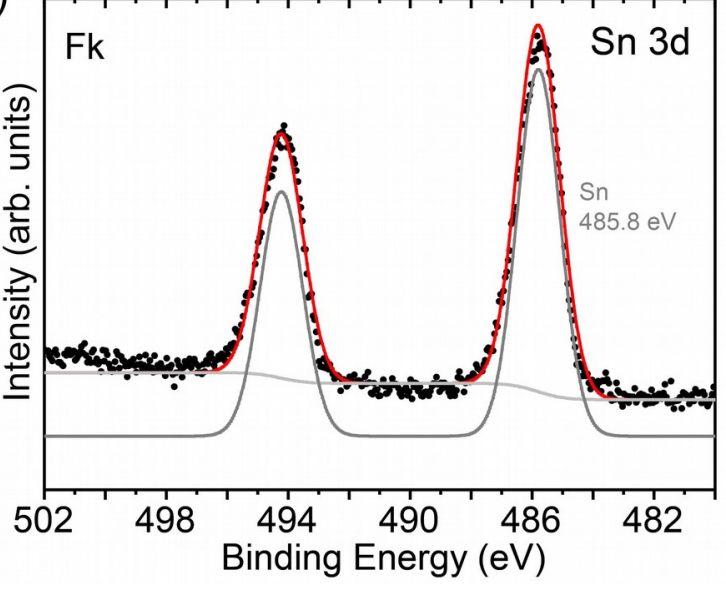

b)

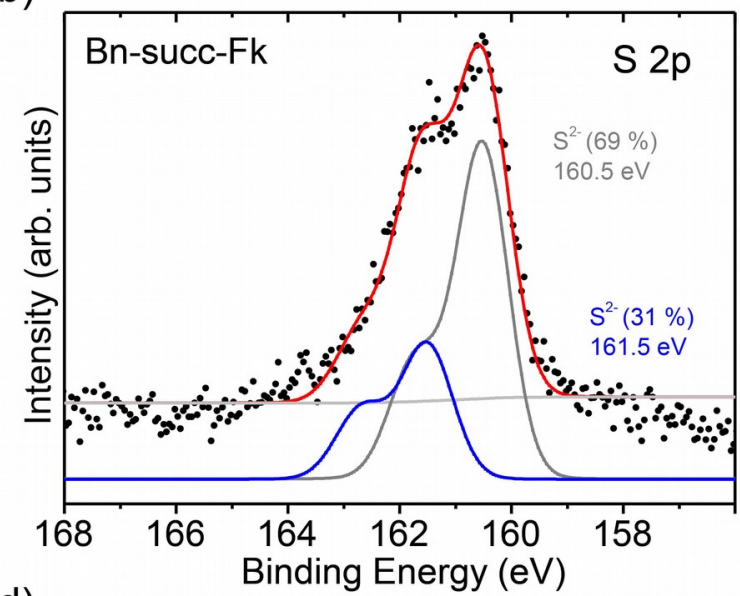

d)
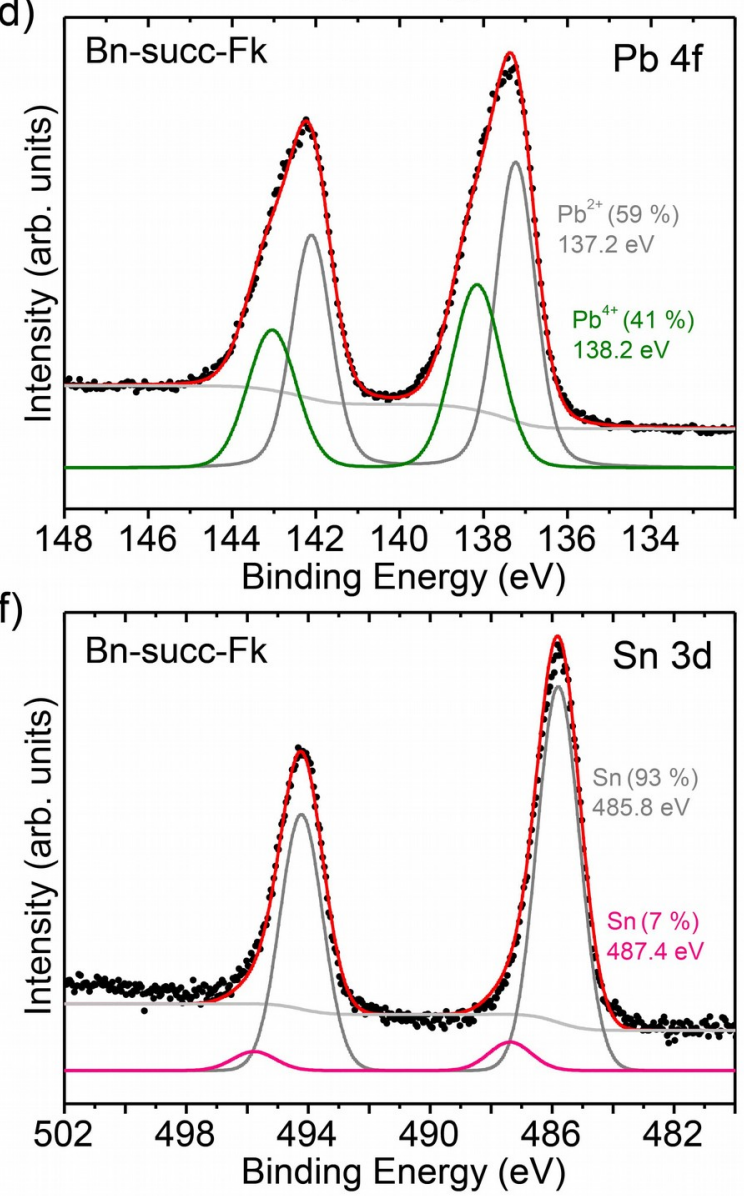

Figure 3. XPS spectra of $\mathrm{S}, \mathrm{Pb}$ and $\mathrm{Sn}$ core levels for $(\mathrm{a}-\mathrm{c})$ the control Fk material and (d-f) Bnsucc-Fk.

As proof-of-principle application of our methodology, a maleimide (TPP-mal) bearing a tetraphenylporphyrin TPP group as a chromophore was employed in the reaction. In this case, a 
clear sign of functionalization is observed in the UV-Vis spectra of the TPP-succ-Fk sample (Figure a). The Soret band of the porphyrin core ( $415 \mathrm{~nm}$ in TPP-mal) is without a doubt responsible for the new absorption band observed in TPP-succ-Fk at $417 \mathrm{~nm}$. Furthermore, perfect match of the Soret band resonances is seen in the 2D PL excitation - emission contour plots depicted in Figure 4c-d and in the PLE spectra (Figure S13-1), suggesting the lack of ground-state interactions across the hybrid interface. The $\mathrm{Q}$ band resonances present in the $470-600 \mathrm{~nm}$ spectral window of the control sample are not so evident in the TPP-succ-Fk excitation spectra. This is most likely a consequence of the low amount of TPP-succ-Fk dispersed in solution rather than a change in electronic structure, as those resonances can be guessed just above the measurement noise level. The lack of ground state interactions is explained by the physical separation between the chromophore and Fk and the truncated $p$-conjugation caused by the maleimide bridge preventing electronic overlap between the OD and 2D entities. In line with TPP-mal, TPP-succ-Fk PL exhibits two bands at 650 and $750 \mathrm{~nm}$. The absence of spectral shifts in the PL spectra (Figure 4b) of TPP-mal and TPP-succ-Fk also seems to point towards negligible dielectric constant changes in the porphyrin surrounding upon its anchorage to Fk.

To gain insights into excited-state interactions across the hybrid interface, time-resolved PL measurements were deployed in TPP-succ-Fk and TPP-mal, observing a clear acceleration of the $\mathrm{PL}$ dynamics in the former. The PL dynamics of TPP-mal detected at $650 \mathrm{~nm}$ displays two decay components of $1 \mathrm{~ns}(5 \%)$ and $10 \mathrm{~ns}$ (95\%) (Figure 4e-f) whilst a new decay component of $0.5 \mathrm{ps}$ $(12 \%)$ is resolved in TPP-succ-Fk. The possible influence of porphyrin aggregation in the observed $\mathrm{PL}$ dynamics changes was foreseen and ruled out based on the identical decay times measured in solutions with lower TPP concentrations decreased by a two decades range, (Figure S13-2). Thus, the fast decay component observed in TPP-succ-Fk is ascribed to excited-state de-activation taking place at the porphyrin/Fk interface. Given the low energy bandgap of $\mathrm{Fk}(0.5 \mathrm{eV})^{20}$ and its $\mathrm{p}$-type character, a type I heterojunction is expected at the interface, with the Fk conduction and valence bands located within the TPP bandgap (2.49 eV HOMO-LUMO energy)..$^{40}$ This scenario would favor a porphyrin-to-Fk energy transfer as the most plausible interaction. Finally, fs-Transient Absorption Spectroscopy (TAS) measurements upon predominant Fk photoexcitation $(387 \mathrm{~nm})$ were conducted, (Figure S14). In these measurements, the differential transmission (DT/T) spectra and dynamics of TPP-succ-Fk (Figure S14-1c, f) are dominated by a broad photoinduced absorption band ascribed to Fk (Figure S14a, d), finding no significant difference between Fk and TPP-succ-Fk (neither in spectral shape nor in temporal dynamics). Global fit analysis of the TAS Fk data applied following a linear cascade model yielded three different relaxation processes with lifetimes $\tau_{1}=200 \mathrm{fs}, \tau_{2}=56 \mathrm{ps}$, and $\tau_{3}=5.4 \mathrm{~ns}$. Similar components are often found in other semiconducting $2 \mathrm{D}$ materials such as $\mathrm{MoS}_{2}$ or $\mathrm{WS}_{2}$, being the sub-ps relaxation ascribed to thermalization of hot excitons, ${ }^{41,42}$ while slower processes are assigned to diffusion, ${ }^{43}$ defect trapping ${ }^{42,44}$ or band-to-band 
recombination. ${ }^{45}$ The lack of significant changes in the TAS spectra and dynamics of Fk and TPPsucc-Fk upon predominant Fk photoexcitation together with the evidences for TPP-succ-Fk PL quenching aforementioned are both compatible with a TPP-to-Fk energy transfer mechanism.

a)

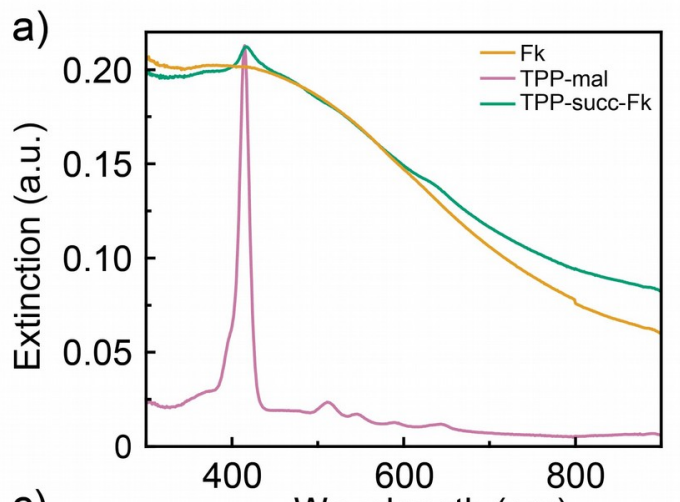

c)

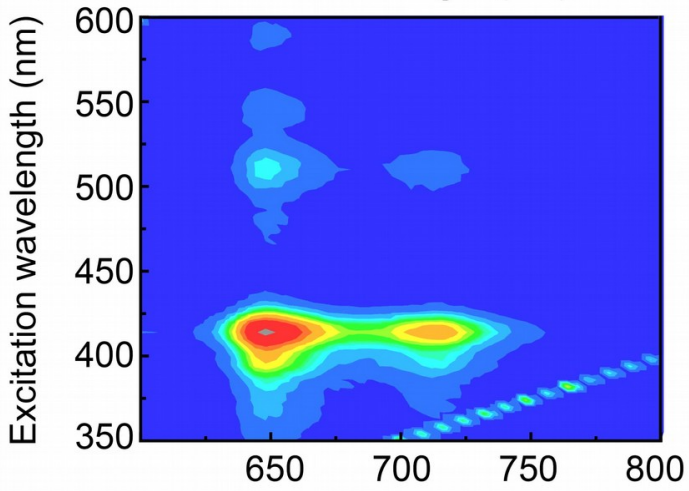

Emission wavelength $(\mathrm{nm})$

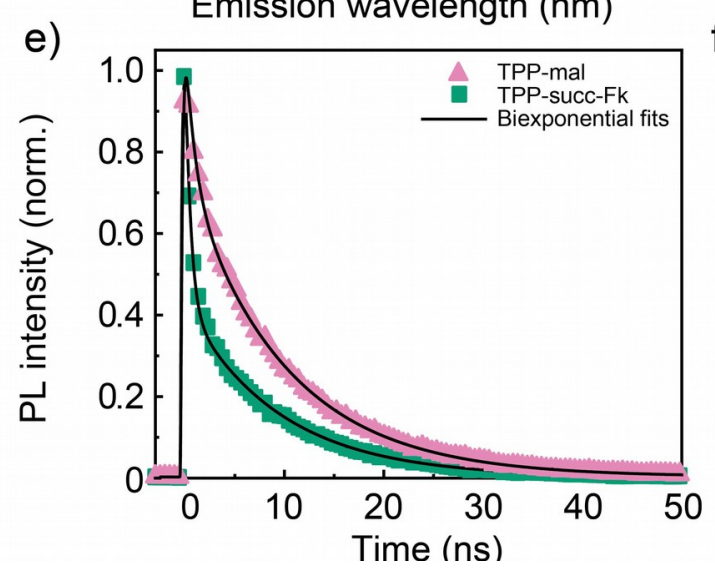

b)

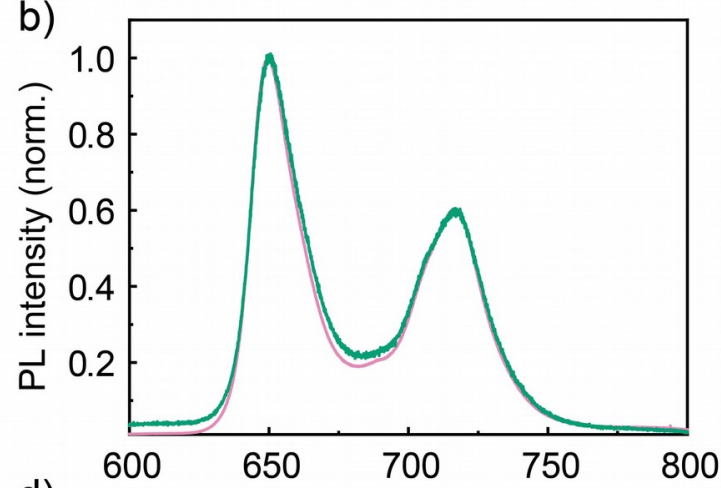

d)

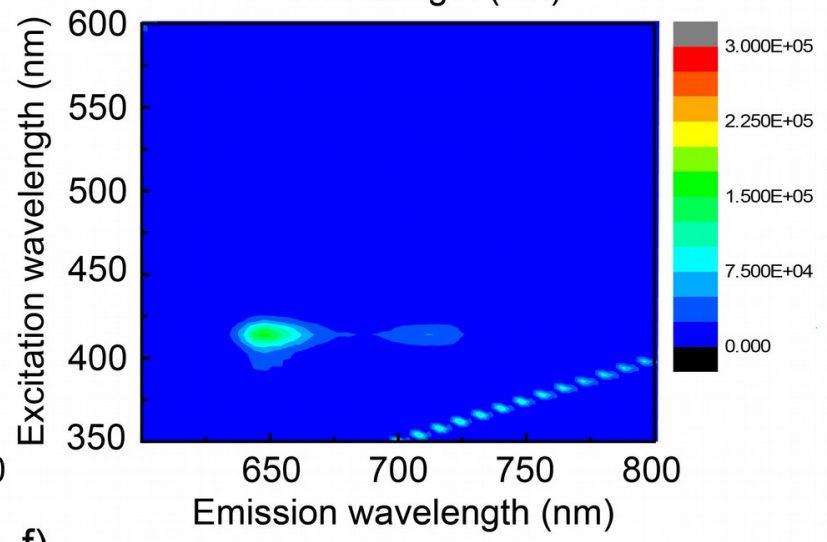

f)

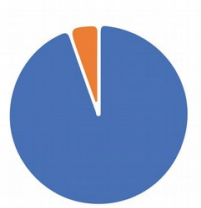

TPP-mal

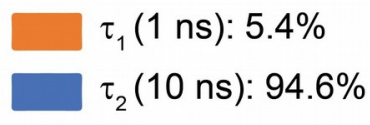

TPP-succ-Fk

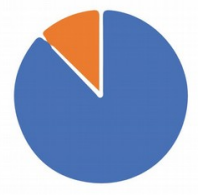

$\tau_{1}(0.5 \mathrm{~ns}): 12.3 \%$

$\tau_{2}(9.5 \mathrm{~ns}): 87.7 \%$

Figure 4. a) UV-Vis spectra of the control Fk sample, tetraphenylporphyrin-derived maleimide (TPP-mal) and TPP-succ-Fk. b) Normalized PL spectra of TPP-mal and TPP-succ-Fk. c) and d) PLE intensity maps of (c) TPP-mal and (d) TPP-succ-Fk, respectively. Rayleigh scattering has not been filtered and the excitation wavelength second order diffraction can be seen as the straight line 
starting at $700 \mathrm{~nm}$ in (c) and (d) $x$ axes. This is a fingerprint of highly scattering samples measured with single grating spectrometers. e) TRPL dynamics of TPP-mal and TPP-succ-Fk photoexcited at $405 \mathrm{~nm}$ and detected at $650 \mathrm{~nm}$ together with bi-exponential fits (straight lines). f) Summary sketch depicting the decay lifetime values and corresponding statistical weights of TPP-mal and TPP-succFk. All spectra were recorded in $\mathrm{iPrOH}$ at room temperature.

In conclusion, we describe how the naturally occurring van der Waals heterostructure Fk can be functionalized covalently making use of the inherent soft electrophilicity of maleimides. ${ }^{33}$ The good quality of the ${ }^{1} \mathrm{H}$ HR-MAS NMR signals allows us to confirm the proposed thiol-maleimide "click" functionalization mechanism beyond reasonable doubt, and serves as an encouraging example for the use of NMR techniques for the characterization of 2D materials. ${ }^{46} \mathrm{HR}$-TEM and XPS confirm that the functionalization is sufficiently mild to respect the native van der Waals heterostructure, occurring preferentially at the $\mathrm{SnS}_{2}$ layer.

Finally, we prove that this reaction can be used to connect functional organic molecules by successfully attaching a porphyrin derivative. The steady-state photophysical properties of the 2D2D-OD mixed-dimensional heterostructure obtained show that the organic chromophore retains its ground state character, whereas an energy transfer process between the porphyrin and the van der Waals heterostructure takes place. 


\section{Associated content}

Supporting Information. Experimental details, characterization and Supporting Figures.

\section{Author Information}

Corresponding Authors:

*juan.cabanillas@imdea.org

*emilio.perez@imdea.org

*@emiliomperezlab

\section{ORCID}

Julia Villalva: 0000-0002-5628-2006

Sara Moreno-Da Silva: 0000-0002-5871-4327

Saül García-Orrit: 0000-0002-3289-7065

Juan Cabanillas González: 0000-0002-9926-3833

Víctor Vega-Mayoral: 0000-0001-8626-0775

Andrés Castellanos-Gómez: 0000-0002-3384-3405

Emerson Giovanelli: 0000-0001-9097-9301

Emilio M. Pérez: 0000-0002-8739-2777

\section{Notes}

The authors declare no competing financial interest.

\section{Acknowledgements}

We acknowledge funding from the European Union (ERC-PoC-842606), MINECO (CTQ201460541-P and CTQ2017-86060-P), and the Comunidad de Madrid and the European Structural Funds for their financial support through FotoArt-CM project (S2018/NMT-4367) and MAD2D-CM S2013/MIT-3007. J. C.-G. acknowledges financial support from the Spanish Ministry of Science, Innovation and Universities (RTI2018-097508-B-I00), and from the Regional Government of Madrid (S2018/NMT4511).. V. V-M acknowledges financial support from the regional government of Madrid "Atracción del talento" program (2019-T2/IND-12737). IMDEA Nanociencia acknowledges support from the "Severo Ochoa" Programme for Centres of Excellence in R\&D (MINECO, grant SEV-20160686). 
1. Y. Liu, N. O. Weiss, X. Duan, H.-C. Cheng, Y. Huang and X. Duan, Nat. Rev. Mater., $2016,1,16042$.

2. K. S. Novoselov, A. Mishchenko, A. Carvalho and A. H. Castro Neto, Science, 2016, 353.

3. A. K. Geim and I. V. Grigorieva, Nature, 2013, 499, 419-425.

4. J. A. Robinson, ACS Nano, 2016, 10, 42-45.

5. P. K. Sahoo, S. Memaran, Y. Xin, L. Balicas and H. R. Gutierrez, Nature, 2018, 553, 63-67.

6. A. Castellanos-Gomez, M. Buscema, R. Molenaar, V. Singh, L. Janssen, H. S. J. van der Zant and G. A. Steele, 2D Mater., 2014, 1, 011002/011001-011002/011008, 011008 pp.

7. R. Frisenda, E. Navarro-Moratalla, P. Gant, D. Perez De Lara, P. Jarillo-Herrero, R. V. Gorbachev and A. Castellanos-Gomez, Chem. Soc. Rev., 2018, 47, 53-68.

8. A. Hirsch and F. Hauke, Angew. Chem., Int. Ed., 2017, 57, 4338-4354.

9. S. Bertolazzi, M. Gobbi, Y. Zhao, P. Samori and C. Backes, Chem. Soc. Rev., 2018, 47, 6845-6888.

10. E. Coronado, C. Martí-Gastaldo, E. Navarro-Moratalla, E. Burzurí, A. Camón and F. Luis, Adv. Mater., 2011, 23, 5021-5026.

11. K. Zhang, T. Zhang, G. Cheng, T. Li, S. Wang, W. Wei, X. Zhou, W. Yu, Y. Sun, P. Wang, D. Zhang, C. Zeng, X. Wang, W. Hu, H. J. Fan, G. Shen, X. Chen, X. Duan, K. Chang and N. Dai, ACS Nano, 2016, 10, 3852-3858.

12. H. Zhang, X. Zhang, C. Liu, S.-T. Lee and J. Jie, ACS Nano, 2016, 10, 5113-5122.

13. M. Massicotte, P. Schmidt, F. Vialla, K. G. Schadler, A. Reserbat-Plantey, K. Watanabe, T. Taniguchi, K. J. Tielrooij and F. H. L. Koppens, Nat. Nanotechnol., 2016, 11, 42-46.

14. M. Long, E. Liu, P. Wang, A. Gao, H. Xia, W. Luo, B. Wang, J. Zeng, Y. Fu, K. Xu, W. Zhou, Y. Lv, S. Yao, M. Lu, Y. Chen, Z. Ni, Y. You, X. Zhang, S. Qin, Y. Shi, W. Hu, D. Xing and F. Miao, Nano Lett., 2016, 16, 2254-2259.

15. G. W. Mudd, S. A. Svatek, L. Hague, O. Makarovsky, Z. R. Kudrynskyi, C. J. Mellor, P. H. Beton, L. Eaves, K. S. Novoselov, Z. D. Kovalyuk, E. E. Vdovin, A. J. Marsden, N. R. Wilson and A. Patane, Adv. Mater., 2015, 27, 3760-3766.

16. L. Wang, X. Zheng, L. Chen, Y. Xiong and H. Xu, Angew. Chem., Int. Ed., 2018, 57, 3454-3458.

17. S. Jiang, J. Shan and K. F. Mak, Nat. Mater., 2018, 17, 406-410.

18. M. J. Kade, D. J. Burke and C. J. Hawker, J. Polym. Sci., Part A: Polym. Chem., 2010, 48, 743-750.

19. C. E. Hoyle and C. N. Bowman, Angew. Chem., Int. Ed., 2010, 49, 1540-1573.

20. A. J. Molina-Mendoza, E. Giovanelli, W. S. Paz, M. A. Nino, J. O. Island, C. Evangeli, L. Aballe, M. Foerster, H. S. J. van der Zant, G. Rubio-Bollinger, N. Agrait, J. J. Palacios, E. M. Perez and A. Castellanos-Gomez, Nature Communications, 2017, 8, 14409.

21. M. Velický, P. S. Toth, A. M. Rakowski, A. P. Rooney, A. Kozikov, C. R. Woods, A. Mishchenko, L. Fumagalli, J. Yin, V. Zólyomi, T. Georgiou, S. J. Haigh, K. S. Novoselov and R. A. W. Dryfe, Nat. Commun., 2017, 8, 14410.

22. R. Gusmão, Z. Sofer, J. Luxa and M. Pumera, Journal of Materials Chemistry A, 2018, 6, 1659016599.

23. S. Gan, Y. Zhao, X. Dai and Y. Xiang, Results in Physics, 2019, 13, 102320.

24. K. Kim, M. Yankovitz, B. Fallahazad, S. Kang, H. C. P. Movva, S. Huang, S. Larentis, C. M. Corbet, T. Taniguchi, K. Watanabe, S. K. Banerjee, B. J. LeRoy and E. Tutuc, Nano Lett., 2016, 16, 1989-1995. 
25. E. Burzuri, M. Vera-Hidalgo, E. Giovanelli, J. Villalva, A. Castellanos-Gomez and E. M. Perez, Nanoscale, 2018, 10, 7966-7970.

26. H. Chen, J. He, C. D. Malliakas, C. C. Stoumpos, A. J. E. Rettie, J.-K. Bao, D. Y. Chung, W.-K. Kwok, C. Wolverton and M. G. Kanatzidis, J. Am. Chem. Soc., 2019, 141, 7544-7553.

27. Y. Niu, J. Villalva, R. Frisenda, G. Sanchez-Santolino, L. Ruiz-González, E. M. Pérez, M. GarcíaHernández, E. Burzurí and A. Castellanos-Gomez, 2D Materials, 2019, 6, 035023.

28. B. Wang, S. P. Zhong, Z. B. Zhang, Z. Q. Zheng, Y. P. Zhang and H. Zhang, Applied Materials Today, 2019, 15, 115-138.

29. J. J. Navarro, F. Calleja, R. Miranda, E. M. Perez and A. L. Vazquez de Parga, Chem. Commun. (Cambridge, U. K.), 2017, 53, 10418-10421.

30. J. J. Navarro, S. Leret, F. Calleja, D. Stradi, A. Black, R. Bernardo-Gavito, M. Garnica, D. Granados, A. L. Vazquez de Parga, E. M. Perez and R. Miranda, Nano Lett., 2016, 16, 355-361.

31. E. Makovicky, M. Dušek, V. Petříček and D. Topa, Am. Mineral., 2011, 96, 1686-1702.

32. K. Ray, A. E. Yore, T. Mou, S. Jha, K. K. H. Smithe, B. Wang, E. Pop and A. K. M. Newaz, ACS Nano, 2017, 11, 6024-6030.

33. M. Vera-Hidalgo, E. Giovanelli, C. Navio and E. M. Perez, J. Am. Chem. Soc., 2019, 141, 3767-3771.

34. A. J. Molina-Mendoza, E. Giovanelli, W. S. Paz, M. Angel Nino, J. O. Island, C. Evangeli, L. Aballe, M. Foerster, H. S. J. van der Zant, G. Rubio-Bollinger, N. Agrait, J. J. Palacios, E. M. Perez and A. Castellanos-Gomez, Nat. Comm., 2017, 8.

35. O. Beckonert, M. Coen, H. C. Keun, Y. Wang, T. M. D. Ebbels, E. Holmes, J. C. Lindon and J. K. Nicholson, Nat. Protoc., 2010, 5, 1019.

36. T. M. Alam and J. E. Jenkins. HR-MAS NMR Spectroscopy in Material Science, Advanced Aspects of Spectroscopy, Ed. Muhammad Akhyar Farrukh, IntechOpen, DOI: 10.5772/48340. Available from: https://www.intechopen.com/books/advanced-aspects-of-spectroscopy/hr-mas-nmr-spectroscopy-inmaterial-science

37. R. Warrass and G. Lippens, J. Org. Chem., 2000, 65, 2946-2950.

38. L. Pauling, J. Am. Chem. Soc., 1932, 54, 988-1003.

39. In fact, adding a third component for the S $2 p$ peak improves the fitting (Fig. S12) but considering our experimental resolution we do not think it is sufficiently justified.

40. P. Zhao, Y. Huang, J. Chen, S. Shao, H. Miao, J. Xia, C. Jia and M. Hua, Journal of Photochemistry and Photobiology, 2020, 3-4, 100014.

41. T. Borzda, C. Gadermaier, N. Vujicic, P. Topolovsek, M. Borovsak, T. Mertelj, D. Viola, C. Manzoni, E. A. A. Pogna, D. Brida, M.-R. Antognazza, F. Scotognella, G. Lanzani, G. Cerullo and D. Mihailovic, Adv. Func. Mater., 2015, 25, 3351-3358.

42. V. Vega-Mayoral, D. Vella, T. Borzda, M. Prijatelj, I. Tempra, E. A. A. Pogna, S. Dal Conte, P. Topolovsek, N. Vujicic, G. Cerullo, D. Mihailovic and C. Gadermaier, Nanoscale, 2016, 8, 5428-5434.

43. N. Kumar, J. He, D. He, Y. Wang and H. Zhao, J. Appl. Phys., 2013, 113, 133702.

44. V. Vega-Mayoral, T. Borzda, D. Vella, M. Prijatelj, E. A. A. Pogna, C. Backes, J. N. Coleman, G. Cerullo, D. Mihailovic and C. Gadermaier, 2D Mater, 2017, 5, 015011.

45. D. Kozawa, R. Kumar, A. Carvalho, K. K. Amara, W. Zhao, S. Wang, M. Toh, R. M. Ribeiro, A. H. Castro Neto, K. Matsuda and G. Eda, Nat Commun, 2014, 5, 4543. 
46. I. A. Vacchi, C. Spinato, J. Raya, A. Bianco and C. Menard-Moyon, Nanoscale, 2016, 8, 1371413721. 